\title{
Inequalities for Products of Polynomials II
}

\author{
I. E. Pritsker* and S. Ruscheweyh ${ }^{\dagger}$
}

Summary. In this paper, we continue the study of inequalities connecting the product of uniform norms of polynomials with the norm of their product, begun in [28]. Asymptotically sharp constants are known for such inequalities over arbitrary compact sets in the complex plane. We show here that such constants can be improved under some natural additional assumptions. Thus we find the best constants for rotationally symmetric sets. In addition, we characterize all sets that allow an improvement in the constant when the number of factors is fixed, and find the improved value.

Mathematics Subject Classification (2000). Primary 30C10; Secondary 30C85, 31A15.

Keywords. Polynomials, products, factors, uniform norm, logarithmic capacity, equilibrium measure, subharmonic function, Fekete points.

\section{The problem and its history}

Let $E$ be a compact set in the complex plane $\mathbb{C}$. For a function $f: E \rightarrow \mathbb{C}$ define the uniform (sup) norm as follows:

$$
\|f\|_{E}=\sup _{z \in E}|f(z)| .
$$

Kneser [18] proved the first sharp inequality for norms of products on $[-1,1]$ (see also Aumann [1] for a preliminary result)

$$
\left\|p_{1}\right\|_{[-1,1]}\left\|p_{2}\right\|_{[-1,1]} \leq K_{\ell, n}\left\|p_{1} p_{2}\right\|_{[-1,1]}, \quad \operatorname{deg} p_{1}=\ell, \operatorname{deg} p_{2}=n-\ell,
$$

where

$$
K_{\ell, n}:=2^{n-1} \prod_{k=1}^{\ell}\left(1+\cos \frac{2 k-1}{2 n} \pi\right) \prod_{k=1}^{n-\ell}\left(1+\cos \frac{2 k-1}{2 n} \pi\right) .
$$

*Research of I.P. was partially supported by the National Security Agency (grant H9823006-1-0055), and by the Alexander von Humboldt Foundation.

${ }^{\dagger}$ S.R. acknowledges partial support from the German-Israeli Foundation (grant G-809234.6/2003), from FONDECYT (grants 1070269 and 7080064) and from DGIP-UTFSM (grant 240862). 
Observe that equality holds in (1.1) for the Chebyshev polynomial

$t(z)=\cos n \arccos z=p_{1}(z) p_{2}(z)$, with a proper choice of the factors $p_{1}(z)$ and $p_{2}(z)$. P. B. Borwein [7] generalized this to the multifactor inequality

$$
\prod_{k=1}^{m}\left\|p_{k}\right\|_{[-1,1]} \leq 2^{n-1} \prod_{k=1}^{\left[\frac{n}{2}\right]}\left(1+\cos \frac{2 k-1}{2 n} \pi\right)^{2}\|p\|_{[-1,1]} .
$$

Note that

$$
2^{n-1} \prod_{k=1}^{\left[\frac{n}{2}\right]}\left(1+\cos \frac{2 k-1}{2 n} \pi\right)^{2} \sim(3.20991 \ldots)^{n} \text { as } n \rightarrow \infty .
$$

For another slight generalization of Kneser's result see Theorem 3.3 below.

A similar inequality for $E=D$, where $D:=\{w:|w| \leq 1\}$ is the closed unit disk, was considered by Gelfond [14, p. 135] in connection with the theory of transcendental numbers:

$$
\prod_{k=1}^{m}\left\|p_{k}\right\|_{D} \leq e^{n}\|p\|_{D}
$$

Mahler [22] later replaced $e$ by 2 :

$$
\prod_{k=1}^{m}\left\|p_{k}\right\|_{D} \leq 2^{n}\|p\|_{D}
$$

It is easy to see that the base 2 cannot be decreased, if $m=n$ and $n \rightarrow \infty$. However, (1.6) has been further improved in two directions. D. W. Boyd [8, 9] showed that, given the number of factors $m$ in (1.6), one has

$$
\prod_{k=1}^{m}\left\|p_{k}\right\|_{D} \leq\left(C_{m}\right)^{n}\|p\|_{D}
$$

where

$$
C_{m}:=\exp \left(\frac{m}{\pi} \int_{0}^{\pi / m} \log \left(2 \cos \frac{t}{2}\right) d t\right)
$$

is asymptotically best possible for each fixed $m$, as $n \rightarrow \infty$. Kroó and Pritsker [19] showed that, for any $m \leq n$,

$$
\prod_{k=1}^{m}\left\|p_{k}\right\|_{D} \leq 2^{n-1}\|p\|_{D}
$$

where equality holds in (1.9) for each $n \in \mathbb{N}$, with $m=n$ and $p(z)=z^{n}-1$.

A natural general problem is to find, for a compact set $E \subset \mathbb{C}$, the smallest constant $M_{E} \in(0, \infty]$, independent of $n$, such that

$$
\prod_{k=1}^{m}\left\|p_{k}\right\|_{E} \leq\left(M_{E}\right)^{n}\|p\|_{E}
$$


holds for arbitrary polynomials $\left\{p_{k}(z)\right\}_{k=1}^{m}$ with complex coefficients, where $p(z)=\prod_{k=1}^{m} p_{k}(z)$ and $n:=\operatorname{deg} p$. The solution of this problem is based on the logarithmic potential theory (cf. [30] and [29]). Let cap $(E)$ be the logarithmic capacity of a compact set $E \subset \mathbb{C}$. For $E$ with $\operatorname{cap}(E)>0$, denote the equilibrium measure of $E$ by $\mu_{E}$. We remark that $\mu_{E}$ is a positive unit Borel measure supported on $\partial E$ (see [30, p. 55]). Define

$$
d_{E}(z):=\max _{t \in E}|z-t|, \quad z \in \mathbb{C},
$$

which is clearly a positive and continuous function in $\mathbb{C}$. It is easy to see that the logarithm of this distance function is subharmonic in $\mathbb{C}$. Furthermore, it has the following integral representation

$$
\log d_{E}(z)=\int \log |z-t| d \sigma_{E}(t), \quad z \in \mathbb{C},
$$

where $\sigma_{E}$ is a positive unit Borel measure in $\mathbb{C}$ with unbounded support, see Lemma 5.1 of [26] and [21]. For further in-depth analysis of the representing measure $\sigma_{E}$, we refer to the recent paper of Gardiner and Netuka [13]. This integral representation is the key fact used by the first author to prove the following result $[26]$.

Theorem 1.1 Let $E \subset \mathbb{C}$ be a compact set, $\operatorname{cap}(E)>0$. Then (1.10) holds with

$$
M_{E}=\frac{\exp \left(\int \log d_{E}(z) d \mu_{E}(z)\right)}{\operatorname{cap}(E)} .
$$

Furthermore, this constant cannot be replaced with a smaller number.

Observe that $M_{E}$ is invariant under similarity transformations of the plane [26].

For the closed unit disk $D$, we have that $\operatorname{cap}(D)=1[30$, p. 84] and that

$$
d \mu_{D}=\frac{d \theta}{2 \pi},
$$

where $d \theta$ is the arclength on $\partial D$. Thus Theorem 1.1 yields

$$
M_{D}=\exp \left(\frac{1}{2 \pi} \int_{0}^{2 \pi} \log d_{D}\left(e^{i \theta}\right) d \theta\right)=\exp \left(\frac{1}{2 \pi} \int_{0}^{2 \pi} \log 2 d \theta\right)=2,
$$

so that we immediately obtain Mahler's inequality (1.6).

If $E=[-1,1]$ then $\operatorname{cap}([-1,1])=1 / 2$ and

$$
d \mu_{[-1,1]}=\frac{d x}{\pi \sqrt{1-x^{2}}}, \quad x \in[-1,1],
$$


which is the Chebyshev (or arcsin) distribution (see [30, p. 84]). Using Theorem 1.1 , we obtain

$$
\begin{aligned}
M_{[-1,1]} & =2 \exp \left(\frac{1}{\pi} \int_{-1}^{1} \frac{\log d_{[-1,1]}(x)}{\sqrt{1-x^{2}}} d x\right)=2 \exp \left(\frac{2}{\pi} \int_{0}^{1} \frac{\log (1+x)}{\sqrt{1-x^{2}}} d x\right) \\
& =2 \exp \left(\frac{2}{\pi} \int_{0}^{\pi / 2} \log (1+\sin t) d t\right) \approx 3.2099123
\end{aligned}
$$

which gives the asymptotic version of Borwein's inequality (1.3)-(1.4).

Considering the above analysis of Theorem 1.1, it is natural to conjecture that the sharp universal bounds for $M_{E}$ are given by

$$
2=M_{D} \leq M_{E} \leq M_{[-1,1]} \approx 3.2099123,
$$

for any bounded non-degenerate continuum $E$, see [27]. We treated this problem in a recent paper [28], where the lower bound $M_{E} \geq M_{D}=2$ is proved for all compact sets $E$, and the upper bound is proved for certain special classes of continua (see also [3]).

It turns out that the upper bound in (1.17) can be decreased under additional assumptions. In particular, Section 2 contains improved bounds of the constant $M_{E}$ for rotationally symmetric sets. The results of Boyd (1.7)-(1.8) suggest that for some sets the constant $M_{E}$ can be replaced by a smaller one, if the number of factors is fixed. We characterize such sets in Section 3, and also find the improved constant. All proofs are given in Section 4.

The problems considered in this paper have many applications in analysis, number theory and computational mathematics. We mention specifically applications in transcendence theory (see Gelfond [14]), and in designing algorithms for factoring polynomials (see Boyd [10] and Landau [20]). A survey of the results involving norms different from the sup norm (e.g., Bombieri norms) can be found in [10]. For polynomials in several variables, see the results of Mahler [23] for the polydisk, of Avanissian and Mignotte [2] for the unit ball in $\mathbb{C}^{k}$. Also, see Beauzamy and Enflo [5], and Beauzamy, Bombieri, Enflo and Montgomery [4] for multivariate polynomials in different norms.

Acknowledgements. This paper was written while the first author was visiting the University of Würzburg as a Humboldt Foundation Fellow. He would like to thank the Department of Mathematics and the Function Theory research group for their hospitality.

\section{Symmetric sets}

Since $D$ has all possible rotational symmetries, one still has $M_{E} \geq 2$ as the best lower estimate for a symmetric set $E$ (see [28]). However, if $E$ has some symmetry, then it is usually possible to improve the upper bounds for $M_{E}$

obtained in the previous section. We show this for sets invariant under the 
cyclic group of rotations generated by the angle $2 \pi / k, k \in \mathbb{N}$, with respect to a fixed point. Translating the set, we can assume that the center of rotation is at the origin.

The following result was proved in [28] (see Corollary 2.3 there). It shows that the constant decreases when the set is enlarged in a certain way. For a compact set $H \subset \mathbb{C}$, we define the unbounded domain $\Omega_{H}$ as the connected component of $\overline{\mathbb{C}} \backslash H$ that contains $\infty$. Note that the boundary $\partial \Omega_{H}$ represents the "outer boundary" of $H$. Consider the compact set

$$
H^{*}:=\bigcap_{z \in \partial \Omega_{H}} D\left(z, d_{H}(z)\right)
$$

Since $H \subset D\left(z, d_{H}(z)\right)$ for any $z \in \mathbb{C}$, we have that $H \subset H^{*}$.

Proposition 2.1 Let $H \subset \mathbb{C}$ be compact, $\operatorname{cap}(H)>0$. If $E$ is a compact set such that $H \subset E \subset H^{*}$, then $M_{E} \leq M_{H}$. Equality holds if and only if $\operatorname{cap}\left(\Omega_{H} \backslash \Omega_{E}\right)=0$.

Define the $k$-star as $S_{k}:=\left\{r e^{2 \pi i l / k}: r \in[0,1], l=1, \ldots, k\right\}$. We need to determine the corresponding set $S_{k}^{*}$, which was defined in Proposition 2.1. It is not difficult to make a geometric observation that we have $S_{k}^{*}=D$ for even $k \in \mathbb{N}$. However, for odd $k \geq 3, S_{k}^{*}$ is obtained by intersecting $k$ congruent disks centered at the roots of unity (the vertices of $S_{k}$ ), whose radius is equal to the distance to the farthest vertex:

$$
S_{k}^{*}=\bigcap_{l=1}^{k} D\left(e^{2 \pi i l / k}, d_{S_{k}}\left(e^{2 \pi i l / k}\right)\right), \quad k \text { is odd, } k \geq 3 .
$$

This is illustrated in Figure 1.

Theorem 2.2 If $S_{k} \subset E \subset S_{k}^{*}, k \geq 2$, then

$$
M_{E} \leq M_{S_{k}}=\exp \left(\frac{k}{\pi} \int_{0}^{\frac{\pi}{k}} \log \left|\int_{t}^{\frac{2 \pi}{k}\left[\frac{k}{2}\right]+\frac{\pi}{k}}\left(e^{i k x}+1\right)\left(e^{i k x}-1\right)^{\frac{2}{k}-1} e^{-i x} d x\right| d t\right)
$$

Several numerical values of $M_{S_{k}}$ are given in the table below, while Figure 2 contains a listplot of $M_{S_{k}}$.

\begin{tabular}{|r|c|}
\hline$k$ & $M_{S_{k}}$ \\
\hline 2 & 3.20991 \\
3 & 2.35653 \\
4 & 2.46834 \\
5 & 2.24386 \\
10 & 2.15730 \\
\hline
\end{tabular}

\begin{tabular}{|r|c|}
\hline$k$ & $M_{S_{k}}$ \\
\hline 20 & 2.07389 \\
30 & 2.04823 \\
40 & 2.03579 \\
50 & 2.02845 \\
100 & 2.01404 \\
\hline
\end{tabular}




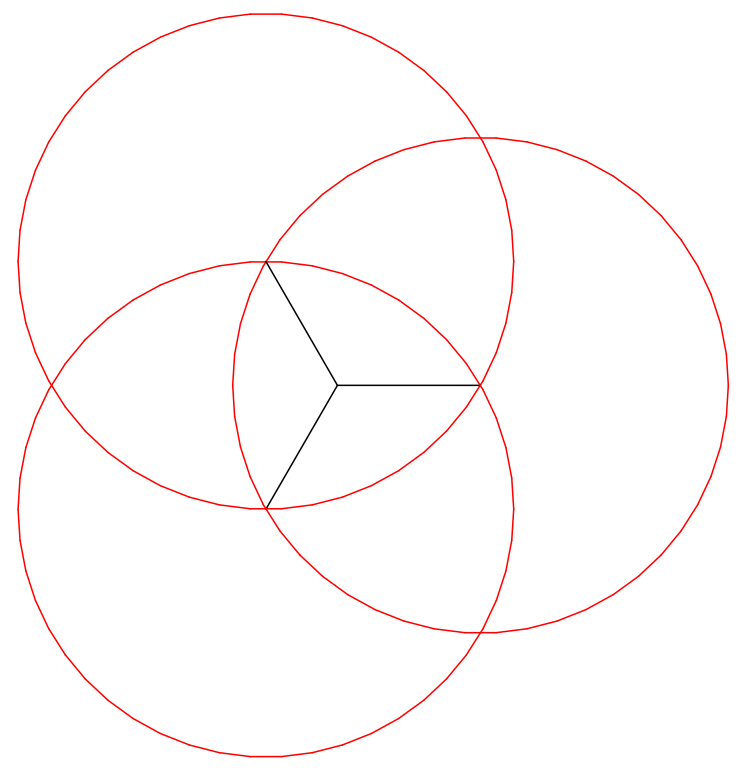

Figure 1: $S_{3}$ and $S_{3}^{*}$.

Next we state a corresponding result for convex sets. Let $P_{k}$ be a regular $k$-gon, with vertices at the $k$ th roots of unity. If $E$ is a compact convex set (not a single point) that is invariant under the rotation by the angle $2 \pi / k, k \in \mathbb{N}, k \geq$ 2 , then we can assume that $P_{k} \subset E \subset D$. Note that $P_{k}^{*}=S_{k}^{*}$ for odd $k \geq 3$. When $k \geq 4$ is even, one obtains that $P_{k}^{*}$ is the intersection of $k$ congruent disks centered at the midpoints of sides of $P_{k}$, with radius equal to the distance to the farthest vertex (see Figure 3):

$P_{k}^{*}=\bigcap_{l=1}^{k} D\left(\frac{e^{2 \pi i l / k}+e^{2 \pi i(l-1) / k}}{2}, d_{P_{k}}\left(\frac{e^{2 \pi i l / k}+e^{2 \pi i(l-1) / k}}{2}\right)\right), \quad k$ is even, $k \geq 4$.

Theorem 2.3 If $P_{k} \subset E \subset P_{k}^{*}, k \geq 2$, then

$$
M_{E} \leq M_{P_{k}}=\exp \left(\frac{k}{\pi} \int_{0}^{\frac{\pi}{k}} \log \left|\int_{t}^{\frac{2 \pi}{k}\left[\frac{k+1}{2}\right]}\left(e^{i k x}-1\right)^{\frac{2}{k}} e^{-i x} d x\right| d t\right) .
$$

Several numerical values of $M_{P_{k}}$ are listed below.

\begin{tabular}{|r|c|}
\hline \multicolumn{1}{|c|}{$k$} & $M_{P_{k}}$ \\
\hline 2 & 3.20991 \\
3 & 2.19901 \\
4 & 2.16503 \\
5 & 2.07882 \\
10 & 2.02405 \\
\hline
\end{tabular}

\begin{tabular}{|r|c|}
\hline \multicolumn{1}{|c|}{$k$} & $M_{P_{k}}$ \\
\hline 20 & 2.00604 \\
30 & 2.00270 \\
40 & 2.00152 \\
50 & 2.00098 \\
100 & 2.00025 \\
\hline
\end{tabular}




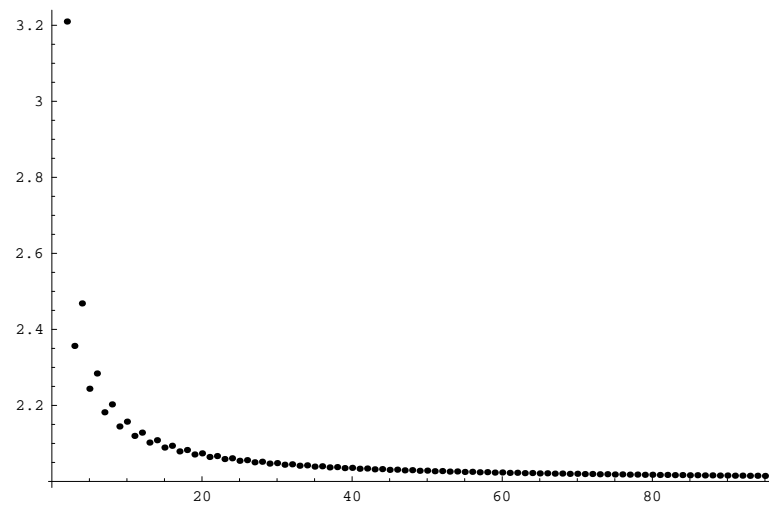

Figure $2: M_{S_{k}}, k=2, \ldots, 100$.

Note that the $M_{P_{k}}$ converge to the limit 2 much more rapidly than the $M_{S_{k}}$, which, of course, is expected.

Observe that $P_{2}$ (as well as $S_{2}$ ) is just a segment, and Theorems 2.2 and 2.3 reduce to Corollary 2.2 of [28] in this case. We conjecture that Theorems 2.2 and 2.3 hold without the inclusion restrictions. Namely, the largest value of the constant $M_{E}$ among all rotationally symmetric sets as defined above is attained for $S_{k}$, while for the convex rotationally symmetric sets $M_{E}$ is maximized for $P_{k}$.

\section{$3 \quad$ Fixed number of factors}

In this section, we explore possible improvements in the constant when the number of factors is fixed. The key results in this direction are due to Boyd $[8,9]$ for the unit disk, see (1.7)-(1.8). For general sets, this question was touched upon in [26], where it was shown that the possibility of improvement essentially depends on the number of extreme points in the set (see Theorem 4.1 in [26]). Specifically, let $\left\{F_{n}(z)\right\}_{n=1}^{\infty}$, $\operatorname{deg} F_{n}=n$, be the Fekete polynomials for the set $E$ (cf. $[29$, p. 155]), where $E \subset \mathbb{C}$ is compact, $\operatorname{cap}(E)>0$. Suppose that there exist points $\left\{\zeta_{l}\right\}_{l=1}^{s}$ such that

$$
d_{E}(z)=\max _{1 \leq l \leq s}\left|z-\zeta_{l}\right| \quad \text { for all } z \in \partial E .
$$

If $m \geq s$ then we can find such factoring for the sequence of Fekete polynomials

$$
F_{n}(z)=\prod_{k=1}^{m} F_{k, n}(z), \quad n \in \mathbb{N},
$$

that

$$
\lim _{n \rightarrow \infty}\left(\frac{\prod_{k=1}^{m}\left\|F_{k, n}\right\|_{E}}{\left\|F_{n}\right\|_{E}}\right)^{1 / n}=M_{E} .
$$




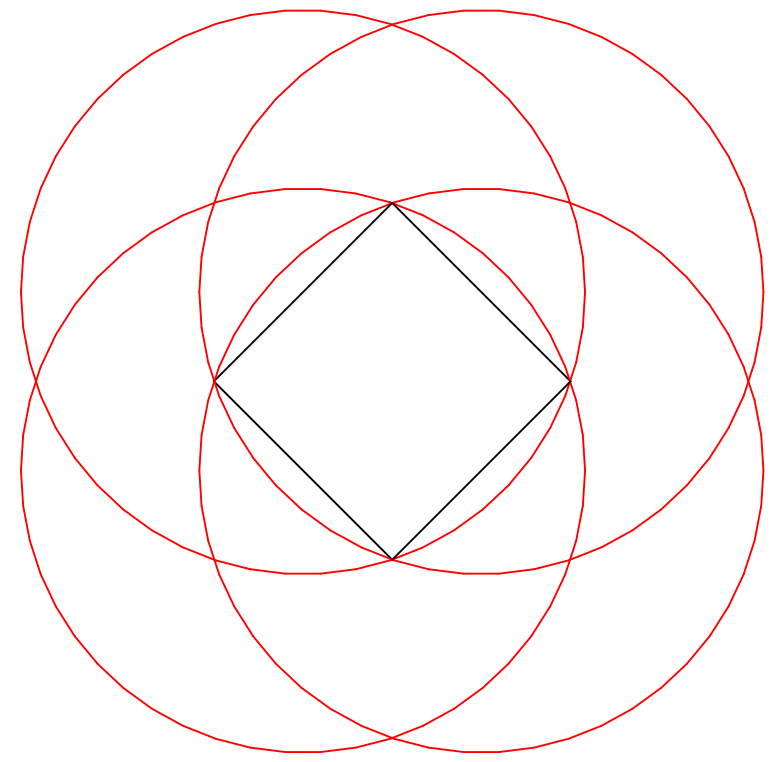

Figure 3: $P_{4}$ and $P_{4}^{*}$.

Hence no improvement is possible in (1.10), for a fixed number of factors $m \geq s$, as $n \rightarrow \infty$. In particular, there is no improvement in constant, for any $m \geq 2$, for such sets as a circular arc of angular measure at most $\pi$ and a segment, cf. (1.1)-(1.3). Also, there is no improvement for any polygon with $s$ vertices, if $m \geq s$.

We give a complete characterization for the possibility of improvement here. A closed set $S \subset E$ is called dominant if

$$
d_{E}(z)=\max _{t \in S}|z-t| \quad \text { for all } z \in \operatorname{supp} \mu_{E} .
$$

This condition is somewhat less restrictive than (3.1), because supp $\mu_{E} \subset \partial \Omega_{E} \subset$ $\partial E$, see [30, p. 79]. Note that if $E$ is the closure of a Jordan domain, then $\operatorname{supp} \mu_{E}=\partial \Omega_{E}=\partial E$. When $E$ has at least one finite dominant set, we define a minimal dominant set $\mathfrak{D}_{E}$ as a dominant set with the smallest number of points $\operatorname{card}\left(\mathfrak{D}_{E}\right)$. Of course, $E$ might not have finite dominant sets at all, in which case we can take any dominant set as the minimal dominant set with $\operatorname{card}\left(\mathfrak{D}_{E}\right)=\infty$, e.g., $\mathfrak{D}_{E}=\partial E$.

Theorem 3.1 Let $E \subset \mathbb{C}$ be compact, $\operatorname{cap}(E)>0$. For arbitrary polynomials $p_{k}, k=1, \ldots, m$, and their product $p, \operatorname{deg}(p)=n$, we have

$$
\prod_{k=1}^{m}\left\|p_{k}\right\|_{E} \leq\left(B_{m}(E)\right)^{n}\|p\|_{E}
$$


where

$$
B_{m}(E):=\max _{c_{k} \in \partial E} \frac{\exp \left(\int \log \max _{1 \leq k \leq m}\left|z-c_{k}\right| d \mu_{E}(z)\right)}{\operatorname{cap}(E)}
$$

cannot be replaced by a smaller constant. Furthermore, if $m<\operatorname{card}\left(\mathfrak{D}_{E}\right)$ then $B_{m}(E)<M_{E}$, while $B_{m}(E)=M_{E}$ for $m \geq \operatorname{card}\left(\mathfrak{D}_{E}\right)$. When $\mathfrak{D}_{E}$ is infinite, $B_{m}(E)<M_{E}$ holds for all $m \in \mathbb{N}, m \geq 2$.

The following result shows that we always have an improvement for smooth sets, which is similar to the disk case.

Corollary 3.2 If $E \subset \mathbb{C}$ is a compact set bounded by finitely many closed $C^{1}$ smooth Jordan curves, then $B_{m}(E)<M_{E}$ for all $m \in \mathbb{N}, m \geq 2$.

On the other hand, we have $B_{m}(E)=M_{E}$ for $m \geq s$ for every polygon with $s$ vertices. Furthermore, not all vertices may belong to the minimal dominating set. For example, if $E$ is an obtuse triangle, then $\mathfrak{D}_{E}$ consists of only two vertices that are the endpoints of the longest side. Hence $B_{m}(E)=M_{E}$ for $m \geq 2$ as in the segment case. Any circular arc of the angular measure at most $\pi$ has its endpoints as the minimal dominating set, which gives $B_{m}(E)=M_{E}$ for $m \geq 2$ here too. However, if the angular measure of this arc is greater than $\pi$, then one immediately obtains that $\mathfrak{D}_{E}$ is infinite, and $B_{m}(E)<M_{E}$ for all $m \geq 2$.

Finding the exact values of $B_{m}(E)$ for general sets is very complicated. Essentially the only known explicit value is due to Boyd for $E=D$, see (1.7)(1.8).

We conclude this section with a simple remark that Kneser's inequality (1.1)(1.2) is true for any compact convex set.

Theorem 3.3 Let $E \subset \mathbb{C}$ be a compact convex set, which is not a single point. For arbitrary polynomials $p_{1}, \operatorname{deg}\left(p_{1}\right)=\ell$, and $p_{2}, \operatorname{deg}\left(p_{2}\right)=n-\ell$, we have

$$
\left\|p_{1}\right\|_{E}\left\|p_{2}\right\|_{E} \leq K_{\ell, n}\left\|p_{1} p_{2}\right\|_{E},
$$

where $K_{\ell, n}$ is given in (1.2).

\section{Proofs}

\subsection{Proofs for Section 2}

Proof of Theorem 2.2.

The inequality $M_{E} \leq M_{S_{k}}$ follows immediately from Proposition 2.1. Thus we only need to find $M_{S_{k}}$. Consider the conformal mapping

$$
\Psi(w)=\int_{1}^{w}\left(s^{k}+1\right)\left(s^{k}-1\right)^{\frac{2}{k}-1} s^{-2} d s=w+\sum_{m=0}^{\infty} \frac{a_{m}}{w^{m}}
$$


of the exterior of the unit disk $\Omega_{D}$ onto the exterior of a $k$-star, which we denote by $S_{k}^{\prime}$ (see [25, pp. 189-196], for example). Note from the symmetry that the $k$-th roots of unity are mapped by $\Psi$ to the origin, and the points obtained by the rotation of these roots of unity by the angle $\pi / k$ are mapped to the vertices of $S_{k}^{\prime}$. Also, it is clear from the expansion of $\Psi$ that the capacity of this $k$-star is equal to 1 . By the invariance with respect to the similarity transformations, we have that $M_{S_{k}^{\prime}}=M_{S_{k}}$.

Recall that the equilibrium measure $\mu_{S_{k}^{\prime}}$ is the harmonic measure of the exterior of $S_{k}^{\prime}$ at $\infty$, which is invariant under the conformal transformation $\Psi$, see $\left[29\right.$, p. 105]. Using this conformal invariance of $\mu_{S_{k}^{\prime}}$, we obtain that

$$
\begin{aligned}
\log M_{S_{k}^{\prime}} & =\int \log d_{S_{k}^{\prime}}(z) d \mu_{S_{k}^{\prime}}(z)=\frac{1}{2 \pi} \int_{0}^{2 \pi} \log d_{S_{k}^{\prime}}\left(\Psi\left(e^{i t}\right)\right) d t \\
& =\frac{k}{\pi} \int_{0}^{\frac{\pi}{k}} \log \left|\Psi\left(e^{i t}\right)-\Psi\left(e^{\frac{2 \pi i}{k}\left[\frac{k}{2}\right]+\frac{\pi i}{k}}\right)\right| d t \\
& =\frac{k}{\pi} \int_{0}^{\frac{\pi}{k}} \log \left|\int_{t}^{\frac{2 \pi}{k}\left[\frac{k}{2}\right]+\frac{\pi}{k}}\left(e^{i k x}+1\right)\left(e^{i k x}-1\right)^{\frac{2}{k}-1} e^{-i x} d x\right| d t .
\end{aligned}
$$

Proof of Theorem 2.3.

The proof of this theorem closely follows the previous one. We obtain the inequality $M_{E} \leq M_{P_{k}}$ from Proposition 2.1. Next we find $M_{P_{k}}$, by introducing the conformal mapping

$$
\Psi(w)=\int_{1}^{w}\left(s^{k}-1\right)^{\frac{2}{k}} s^{-2} d s=w+\sum_{m=0}^{\infty} \frac{a_{m}}{w^{m}}
$$

of $\Omega_{D}$ onto the exterior of a regular $k$-gon denoted by $P_{k}^{\prime}[25$, p. 196]. The $k$-th roots of unity are mapped by $\Psi$ to the vertices of $P_{k}^{\prime}$. Also, it is clear from the expansion of $\Psi$ that the capacity of $P_{k}^{\prime}$ is equal to 1 . Hence we obtain that

$$
\begin{aligned}
\log M_{P_{k}} & =\log M_{P_{k}^{\prime}}=\int \log d_{P_{k}^{\prime}}(z) d \mu_{P_{k}^{\prime}}(z)=\frac{1}{2 \pi} \int_{0}^{2 \pi} \log d_{P_{k}^{\prime}}\left(\Psi\left(e^{i t}\right)\right) d t \\
& =\frac{k}{\pi} \int_{0}^{\frac{\pi}{k}} \log \left|\Psi\left(e^{i t}\right)-\Psi\left(e^{\frac{2 \pi i}{k}\left[\frac{k+1}{2}\right]}\right)\right| d t \\
& =\frac{k}{\pi} \int_{0}^{\frac{\pi}{k}} \log \left|\int_{t}^{\frac{2 \pi}{k}\left[\frac{k+1}{2}\right]}\left(e^{i k x}-1\right)^{\frac{2}{k}} e^{-i x} d x\right| d t .
\end{aligned}
$$

\subsection{Proofs for Section 3}

Proof of Theorem 3.1. 
For any $k=1, \ldots, m$, there exists $c_{k} \in \partial E$ such that

$$
\left\|p_{k}\right\|_{E}=\left|p_{k}\left(c_{k}\right)\right| .
$$

Applying Lemma 5.1 of [26] to the set $\left\{c_{k}\right\}_{k=1}^{m}$, we obtain for the function

$$
u_{m}(z):=\max _{1 \leq k \leq m}\left|z-c_{k}\right|, \quad z \in \mathbb{C},
$$

that

$$
\log u_{m}(z)=\int \log |z-t| d \sigma_{m}(t), \quad z \in \mathbb{C},
$$

where $\sigma_{m}$ is a probability measure on $\mathbb{C}$. If $Z_{k}$ is the set of zeros of $p_{k}(z)$ (counted according to multiplicities), $k=1, \ldots, m$, then

$$
\begin{aligned}
\sum_{k=1}^{m} \log \left\|p_{k}\right\|_{E} & =\sum_{k=1}^{m} \log \left|p_{k}\left(c_{k}\right)\right|=\sum_{k=1}^{m} \sum_{z \in Z_{k}} \log \left|c_{k}-z\right| \leq \sum_{k=1}^{m} \sum_{z \in Z_{k}} \log u_{m}(z) \\
& =\sum_{z \in \bigcup_{k=1}^{m} Z_{k}} \int \log |z-t| d \sigma_{m}(t)=\int \log |p(t)| d \sigma_{m}(t)
\end{aligned}
$$

Using the Bernstein-Walsh lemma [29, p. 156], we proceed further as follows:

$$
\begin{aligned}
\sum_{k=1}^{m} \log \left\|p_{k}\right\|_{E} & \leq \int\left(\log \|p\|_{E}+n g_{E}(t, \infty)\right) d \sigma_{m}(t) \\
& =\int\left(\log \|p\|_{E}+n \log \frac{1}{\operatorname{cap}(E)}+n \int \log |z-t| d \mu_{E}(z)\right) d \sigma_{m}(t) \\
& =\log \|p\|_{E}+n \log \frac{1}{\operatorname{cap}(E)}+n \iint \log |z-t| d \mu_{E}(z) d \sigma_{m}(t) \\
& =\log \|p\|_{E}+n \log \frac{1}{\operatorname{cap}(E)}+n \iint \log |z-t| d \sigma_{m}(t) d \mu_{E}(z) \\
& =\log \|p\|_{E}+n \log \frac{1}{\operatorname{cap}(E)}+n \int \log u_{m}(z) d \mu_{E}(z),
\end{aligned}
$$

where we changed the order of integration by Fubini's theorem. It follows from the above estimate that

$$
\prod_{k=1}^{m}\left\|p_{k}\right\|_{E} \leq\left(\frac{\exp \left(\int \log u_{m}(z) d \mu_{E}(z)\right)}{\operatorname{cap}(E)}\right)^{n}\|p\|_{E} .
$$

Note that $\log u_{m}(z)$ is a continuous function of $c_{k} \in \partial E, k=1, \ldots, m$. Hence $\exp \left(\int \log u_{m}(z) d \mu_{E}(z)\right)$ is also continuous for $c_{k} \in \partial E, k=1, \ldots, m$, and attains its maximum on $(\partial E)^{m}$ for some set $c_{k}^{*} \in \partial E, k=1, \ldots, m$. Thus (3.5)(3.6) are proved. We now show that $B_{m}(E)$ cannot be replaced by a smaller constant, by following the proof of Theorem 4.1 in [26]. Let

$$
u_{m}^{*}(z):=\max _{1 \leq k \leq m}\left|z-c_{k}^{*}\right|, \quad z \in \mathbb{C} .
$$


For the $n$-th Fekete points $\left\{a_{l, n}\right\}_{l=1}^{n}$ of $E$, consider the Fekete polynomials [29, pp. 152-155]

$$
F_{n}(z)=\prod_{l=1}^{n}\left(z-a_{l, n}\right), \quad n \in \mathbb{N} .
$$

We define a subset $\mathcal{F}_{k, n} \subset\left\{a_{l, n}\right\}_{l=1}^{n}$, associated with the point $c_{k}^{*}, k=1, \ldots, m$, so that $a_{l_{0}, n} \in \mathcal{F}_{k, n}$ for some $1 \leq l_{0} \leq n$ if

$$
u_{m}^{*}\left(a_{l_{0}, n}\right)=\left|a_{l_{0}, n}-c_{k}^{*}\right| .
$$

In the case that (4.6) holds for more than one $c_{k}^{*}$, we assign $a_{l_{0}, n}$ to only one set $\mathcal{F}_{k, n}$, to avoid an overlap of these sets. It is then clear that, for any $n \in \mathbb{N}$,

$$
\bigcup_{k=1}^{m} \mathcal{F}_{k, n}=\left\{a_{l, n}\right\}_{l=1}^{n} \quad \text { and } \quad \mathcal{F}_{k_{1}, n} \bigcap \mathcal{F}_{k_{2}, n}=\emptyset, k_{1} \neq k_{2}
$$

The desired factors of $F_{n}(z)$ are defined as

$$
F_{k, n}(z):=\prod_{a_{l, n} \in \mathcal{F}_{k, n}}\left(z-a_{l, n}\right), \quad k=1, \ldots, m
$$

so that

$$
\left\|F_{k, n}\right\|_{E} \geq \prod_{a_{l, n} \in \mathcal{F}_{k, n}}\left|c_{k}^{*}-a_{l, n}\right|=\prod_{a_{l, n} \in \mathcal{F}_{k, n}} u_{m}^{*}\left(a_{l, n}\right), \quad k=1, \ldots, m .
$$

It follows by Lemma 5.3 of [26] (see also [29, p. 159]) that

$$
\begin{aligned}
\liminf _{n \rightarrow \infty}\left(\prod_{k=1}^{m}\left\|F_{k, n}\right\|_{E}\right)^{1 / n} & \geq \lim _{n \rightarrow \infty}\left(\prod_{l=1}^{n} u_{m}^{*}\left(a_{l, n}\right)\right)^{1 / n} \\
& =\lim _{n \rightarrow \infty} \exp \left(\frac{1}{n} \sum_{k=1}^{n} \log u_{m}^{*}\left(a_{k, n}\right)\right) \\
& =\exp \left(\int \log u_{m}^{*}(z) d \mu_{E}(z)\right) .
\end{aligned}
$$

In addition, we have that $\lim _{n \rightarrow \infty}\left\|F_{n}\right\|_{E}^{1 / n}=\operatorname{cap}(E)[29$, p. 155], which gives

$$
\liminf _{n \rightarrow \infty}\left(\frac{\prod_{k=1}^{m}\left\|F_{k, n}\right\|_{E}}{\left\|F_{n}\right\|_{E}}\right)^{1 / n} \geq B_{m}(E) .
$$

Since $u_{m}(z) \leq d_{E}(z)$ for any $z \in \mathbb{C}$, we immediately obtain that $B_{m}(E) \leq$ $M_{E}$. Suppose that $m<\operatorname{card}\left(\mathfrak{D}_{E}\right)$. Then there is $z_{0} \in \operatorname{supp} \mu_{E}$ such that $u_{m}^{*}\left(z_{0}\right)<d_{E}\left(z_{0}\right)$. As both functions are continuous, the same strict inequality holds in a neighborhood of $z_{0}$, so that $\int \log u_{m}^{*}(z) d \mu_{E}(z)<\int \log d_{E}(z) d \mu_{E}(z)$ and $B_{m}(E)<M_{E}$. When $\mathfrak{D}_{E}$ is infinite, this argument gives that $B_{m}(E)<$ 
$M_{E}, m \geq 2$. Assume now that $\mathfrak{D}_{E}$ is finite and that $m \geq \operatorname{card}\left(\mathfrak{D}_{E}\right)$. Then $u_{m}^{*}(z)=d_{E}(z)$ for all $z \in \operatorname{supp} \mu_{E}$, because one of the possible choices of the points $\left\{c_{k}\right\}_{k=1}^{m} \subset \partial E$ includes points of the set $\mathfrak{D}_{E}$. It is immediate that $\int \log u_{m}^{*}(z) d \mu_{E}(z)=\int \log d_{E}(z) d \mu_{E}(z)$ and $B_{m}(E)=M_{E}$ in this case.

Proof of Corollary 3.2.

We need to show that the minimal dominant set is infinite, hence the result follows from Theorem 3.1. Suppose to the contrary that $\mathfrak{D}_{E}=\left\{\zeta_{l}\right\}_{l=1}^{s}$ is finite. Let $J \subset \partial \Omega_{E}$ be a smooth closed Jordan curve. Then $J \subset \operatorname{supp} \mu_{E}=\partial \Omega_{E}[30$, p. 79]. Define

$$
J_{l}:=\left\{z \in J: d_{E}(z)=\left|z-\zeta_{l}\right|\right\}, \quad l=1, \ldots, s .
$$

It is clear that $J=\cup_{l=1}^{s} J_{l}$. Observe that the segment $\left[z, \zeta_{l}\right], z \in J_{l}$, is orthogonal to $\partial \Omega_{E}$ at $\zeta_{l}$. Hence each $J_{l}$ is contained in the normal line to $\partial \Omega_{E}$ at $\zeta_{l}, l=$ $1, \ldots, s$. We thus obtain that $J$ is contained in a union of straight lines, so that $J$ cannot have a continuously turning tangent, which contradicts the smoothness assumption.

Proof of Theorem 3.3.

Let $z_{1}, z_{2} \in \partial E$ be such that $\left\|p_{1}\right\|_{E}=\left|p_{1}\left(z_{1}\right)\right|$ and $\left\|p_{2}\right\|_{E}=\left|p_{2}\left(z_{2}\right)\right|$. Since $E$ is convex, we have that $I:=\left[z_{1}, z_{2}\right] \subset E$ and

$$
\frac{\left\|p_{1}\right\|_{E}\left\|p_{2}\right\|_{E}}{\left\|p_{1} p_{2}\right\|_{E}} \leq \frac{\left|p_{1}\left(z_{1}\right) \| p_{2}\left(z_{2}\right)\right|}{\left\|p_{1} p_{2}\right\|_{I}} \leq \frac{\left\|p_{1}\right\|_{I}\left\|p_{2}\right\|_{I}}{\left\|p_{1} p_{2}\right\|_{I}} \leq K_{l, n}
$$

by Kneser's inequality.

\section{References}

[1] G. Aumann, Satz über das Verhalten von Polynomen auf Kontinuen, Sitz. Preuss. Akad. Wiss. Phys.-Math. Kl. (1933), 926-931.

[2] V. Avanissian and M. Mignotte, A variant of an inequality of Gel'fond and Mahler, Bull. London Math. Soc. 26 (1994), 64-68.

[3] A. Baernstein II, R. S. Laugesen, and I. E. Pritsker, Moment inequalities for equilibrium measures, Pure Appl. Math. Q. (to appear)

[4] B. Beauzamy, E. Bombieri, P. Enflo and H. L. Montgomery, Products of polynomials in many variables, J. Number Theory 36 (1990), 219-245.

[5] B. Beauzamy and P. Enflo, Estimations de produits de polynômes, J. Number Theory 21 (1985), 390-413. 
[6] C. Benitez, Y. Sarantopoulos and A. Tonge, Lower bounds for norms of products of polynomials, Math. Proc. Cambridge Philos. Soc. 124 (1998), 395-408.

[7] P. B. Borwein, Exact inequalities for the norms of factors of polynomials, Can. J. Math. 46 (1994), 687-698.

[8] D. W. Boyd, Two sharp inequalities for the norm of a factor of a polynomial, Mathematika 39 (1992), 341-349.

[9] D. W. Boyd, Sharp inequalities for the product of polynomials, Bull. London Math. Soc. 26 (1994), 449-454.

[10] D. W. Boyd, Large factors of small polynomials, Contemp. Math. 166 (1994), 301-308.

[11] D. W. Boyd, Bounds for the height of a factor of a polynomial in terms of Bombieri's norms: I. The largest factor, J. Symbolic Comp. 16 (1993), 115-130.

[12] D. W. Boyd, Bounds for the height of a factor of a polynomial in terms of Bombieri's norms: II. The smallest factor, J. Symbolic Comp. 16 (1993), 131-145.

[13] S. J. Gardiner and I. Netuka, Potential theory of the farthest-point distance function, J. Anal. Math. 101 (2006), 163-177.

[14] A. O. Gelfond, Transcendental and Algebraic Numbers, Dover, New York, 1960.

[15] P. Glesser, Nouvelle majoration de la norme des facteurs d'un polynôme, C. R. Math. Rep. Acad. Sci. Canada 12 (1990), 224-228.

[16] G. M. Goluzin, Geometric Theory of Functions of a Complex Variable, Vol. 26 of Translations of Mathematical Monographs, Amer. Math. Soc., Providence, R.I., 1969.

[17] A. Granville, Bounding the coefficients of a divisor of a given polynomial, Monatsh. Math. 109 (1990), 271-277.

[18] H. Kneser, Das Maximum des Produkts zweies Polynome, Sitz. Preuss. Akad. Wiss. Phys.-Math. Kl. (1934), 429-431.

[19] A. Kroó and I. E. Pritsker, A sharp version of Mahler's inequality for products of polynomials, Bull. London Math. Soc. 31 (1999), 269-278.

[20] S. Landau, Factoring polynomials quickly, Notices Amer. Math. Soc. 34 (1987), 3-8.

[21] R. S. Laugesen and I. E. Pritsker, Potential theory of the farthest-point distance function, Can. Math. Bull. 46 (2003), 373-387. 
[22] K. Mahler, An application of Jensen's formula to polynomials, Mathematika 7 (1960), 98-100.

[23] K. Mahler, On some inequalities for polynomials in several variables, J. London Math. Soc. 37 (1962), 341-344.

[24] M. Mignotte, Some useful bounds, In "Computer Algebra, Symbolic and Algebraic Computation" (B. Buchberger et al., eds.), pp. 259-263, SpringerVerlag, New York, 1982.

[25] Z. Nehari, Conformal Mapping, McGraw-Hill Co, New York, 1952.

[26] I. E. Pritsker, Products of polynomials in uniform norms, Trans. Amer. Math. Soc. 353 (2001), 3971-3993.

[27] I. E. Pritsker, Norms of products and factors of polynomials, in "Number Theory for the Millennium III," M. A. Bennett, B. C. Berndt, N. Boston, H. Diamond, A. J. Hildebrand and W. Philipp (eds.), pp. 173-189, A K Peters, Ltd., Natick, 2002.

[28] I. E. Pritsker and S. Ruscheweyh, Inequalities for products of polynomials I, Math. Scand. (to appear)

[29] T. Ransford, Potential Theory in the Complex Plane, Cambridge University Press, Cambridge, 1995.

[30] M. Tsuji, Potential Theory in Modern Function Theory, Chelsea Publ. Co., New York, 1975.

I. E. Pritsker

Department of Mathematics

401 Mathematical Sciences

Oklahoma State University

Stillwater, OK 74078-1058

U.S.A.

e-mail: igor@math.okstate.edu

S. Ruscheweyh

Institut für Mathematik

Universität Würzburg

Am Hubland

97074 Würzburg

Germany

e-mail: ruscheweyh@mathematik.uni-wuerzburg.de 